\title{
NANOSCALE GAP FABRICATION AND INTEGRATION OF CARBON NANOTUBES BY MICROMACHINING
}

\author{
Jaehyun Chung, Junghoon Lee, Rodney S. Ruoff, and Wing Kam Liu \\ Mechanical Engineering, Northwestern University \\ Evanston, IL, 60208
}

\begin{abstract}
This paper presents new techniques for fabricating nanoscale gaps based on micro-lithography and the assembly of carbon nanotubes(CNTs) across these gaps. A sharp nanoscale gap was fabricated by the controlled over-etching of an Al thin film. The etching process was monitored by measured resistance to fabricate a nanoscale gap $(\sim 20 \mathrm{~nm})$ was fabricated. It was found that the fluctuation of the etching rate during the final minutes was so uniform that we could rely on timed etching to obtain the nanoscale gap. A round-shaped gap was also created by reversing a bridge pattern with a lift-off process. The smallest gap created by this method was $150 \mathrm{~nm}$. AC electrophoresis was used to deposit CNTs dispersed in a liquid across the prepared gaps. A highly oriented deposition was obtained across a round gap. Compared with chemical vapor deposition (CVD) methods, the suggested deposition steps can be performed at a room temperature in an open environment, thus providing more process freedom. Various configurations for device application and fundamental experiments can be conceived using the proposed method.
\end{abstract}

\section{INTRODUCTION}

Since their discovery in 1991[1], carbon nanotubes (CNTs) have been studied for many applications due to their unique characteristics. For example, some CNTs are a few micrometers long with the diameter of 1 25nm. A remarkably robust nanoscale composition with such a high aspect ratio can enable high strength structures [2]. Also, the outstanding electrical, mechanical, and chemical characteristics create possibilities for various applications in nanoscale sensing and actuation [3-6]. For device applications, highly ordered nanoscale structures of CNTs often need to be integrated with microscale systems for input/output functions. Material testing, for example, requires a movable stage fabricated by micromachining [7]. Figure 1 shows an example configuration

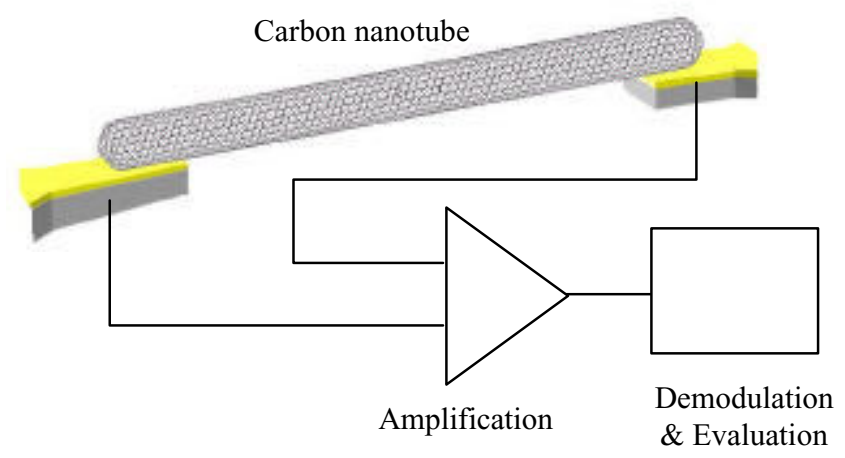

Figure 1. Carbon-nanotube-based sensor

*Travel support has been generously provided by the Transducers Research Foundation and by the DARPA MEMS and DARPA BioFlips programs. for chemical sensing by electromechanical transduction. The CNT deposited in the gap is driven into resonance by either an electrostatic or electromagnetic force. The resonance characteristic can be readily altered by an extremely small amount, e.g, countable number, of molecular deposits by reaction with a functionalized surface. For these kinds of device applications, nanoscale gaps $(50-500 \mathrm{~nm})$ are essential to mechanically support CNTs, to provide actuation, and to receive the electro-mechanical response of CNTs.

It has been shown that CNTs could be positioned by growing them across a gap [8]. This process, however, needed very accurately patterned catalyst sites and a high temperature over $900^{\circ} \mathrm{C}$. Also, an electric field had to be provided to control the direction of deposition, which raises the issue of providing a voltage on a sample inside a high-temperature, closed chamber. This paper presents new techniques for fabricating nanoscale gaps based on micro-lithography and assembling CNTs across these gaps at a room temperature in an open environment. Unlike serial process such as electron beam lithography, the proposed nano-gap fabrication method can be readily combined with current micromachining techniques, and applied to batch production. CNTs dispersed in a solution were directionally deposited across these gaps guided by an electric field.

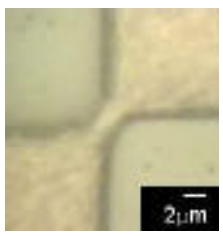

(a)

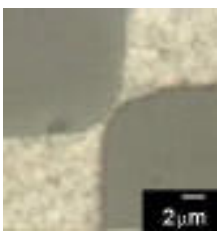

(b)

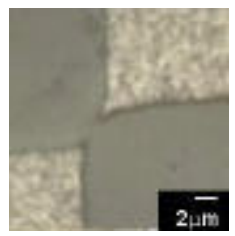

(c)
Figure 2. Sharp gap fabrication by Al etching (a) initial etching (b) additional etching (c) necking and gap creation

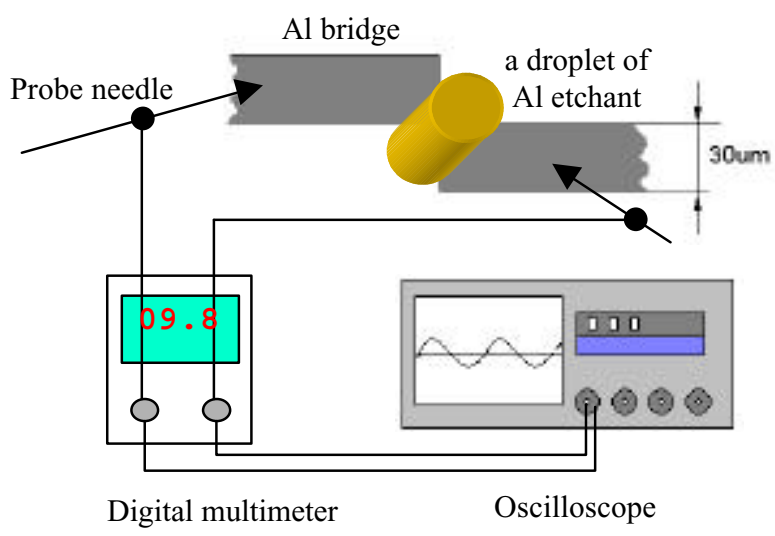

Figure 3. The monitoring of Al etching 


\section{FABRICATION PROCESS}

Two kinds of gaps - a sharp gap and a round gap - were fabricated in this paper. A sharp gap was fabricated by a two-step etching of $\mathrm{Al}$, and a round gap was made by reversing an $\mathrm{Al}$ bridge pattern with a lift-off process.

Figure 2 shows the two-step etching process used to create a sharp gap. $\mathrm{Al}(1200 \AA)$ was deposited on a $\mathrm{SiO}_{2}$ layer and patterned after lithography with $\sim 4 \mu \mathrm{m}$ pattern overlap. The $\mathrm{Al}$ layer was initially etched by a wet etchant at $23{ }^{\circ} \mathrm{C}$ until a $\sim 2 \mu \mathrm{m}$ wide pattern remained due to undercutting (a). After the initial etching and rinsing, a drop of $\mathrm{Al}$ etchant was used to further etch the bridge-shaped pattern (b). Figure 3 shows an experimental setup to monitor the etching rate with an ohm-meter. Subsequent necking occurred, and a sharp gap was created (c).

It was found that even though the total etching time to create a gap may vary much, the fluctuation of etching rate during the final ten minutes is relatively small. We speculate that this is attributed to the reaction-limited process due to the decrease in etching product shortly before the gap is opened. Figure 4 shows the reciprocal of the etching rate (rate of decreasing resistance) determined by measured resistance. The etching rate was not consistent until the value was over $5 \mathrm{~min} / \Omega$, but its variation afterward was small enough to be considered uniform.

The gap size thus could be controlled by the etching time

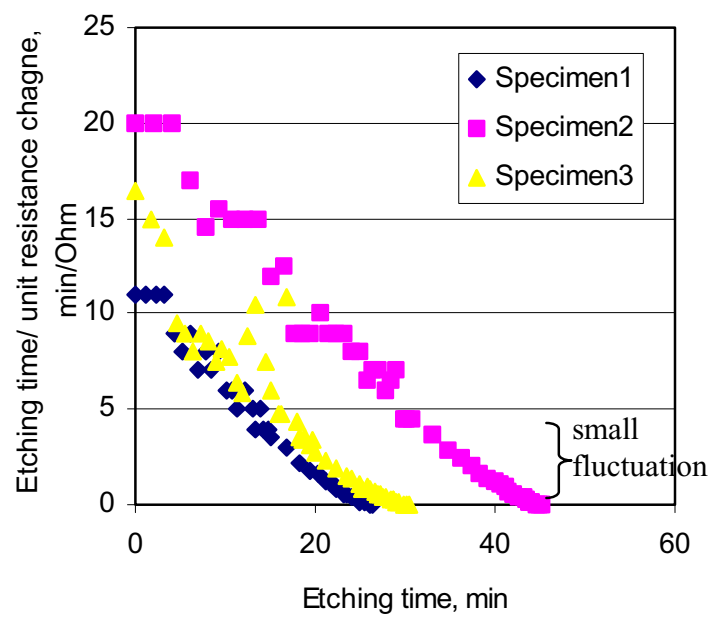

Figure 4. Etching time vs. etching time/unit resistance change $\left(R^{-1}\right)$

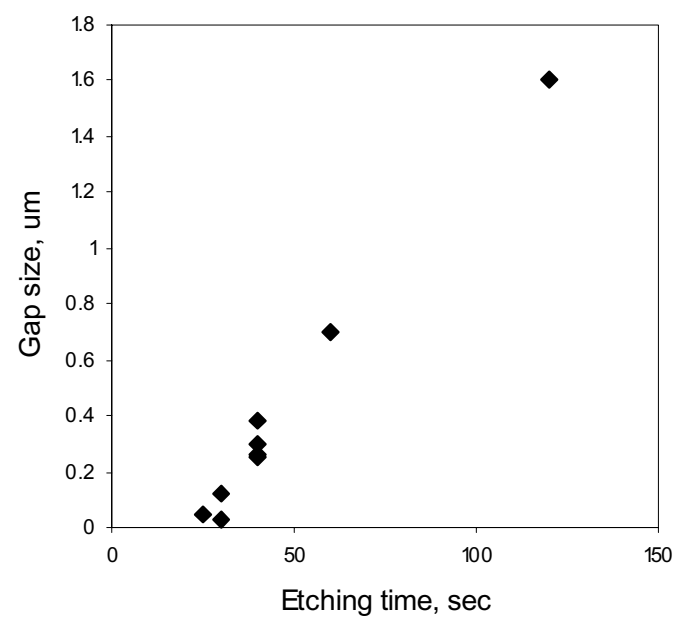

Figure 5. Terminal etching time vs. the size of a sharp gap

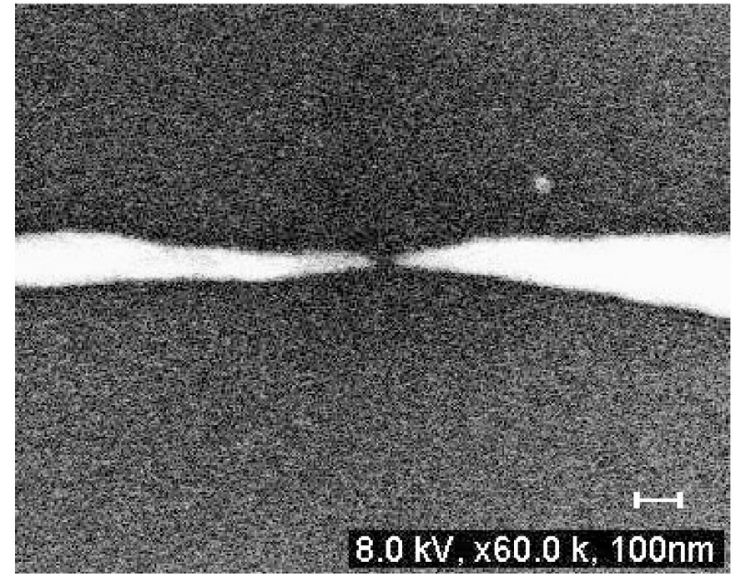

Figure 6. The sharp gap of $50 \mathrm{~nm}$

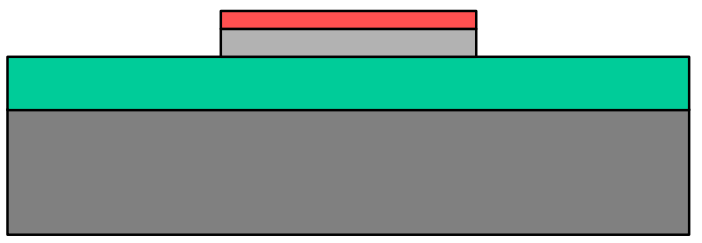

(a) $\mathrm{LPCVD} \mathrm{Si}_{3} \mathrm{~N}_{4}$ and Al patterning

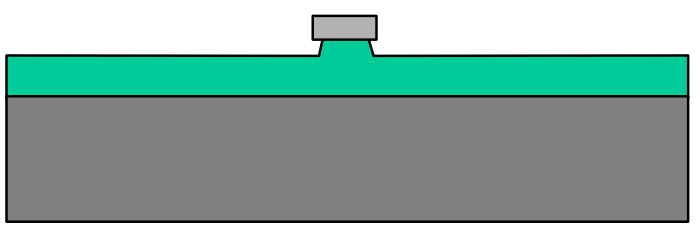

(b) Al horizontal etching and Isotropic RIE

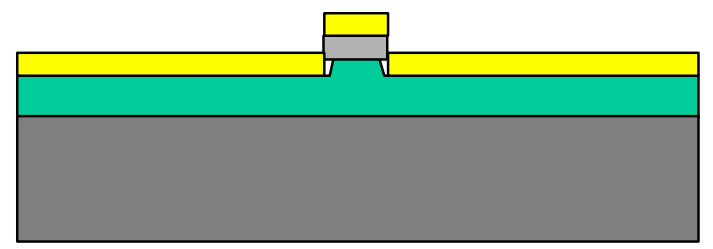

(c) Cr deposition

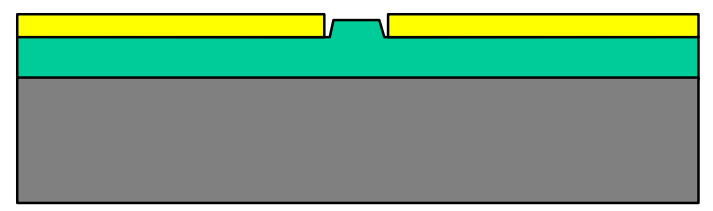

(d) Al lift-off in Al etchant

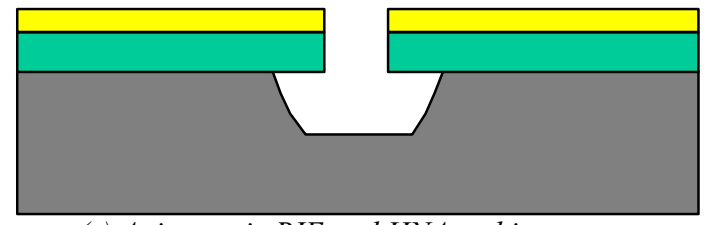

(e) Anisotropic RIE and HNA etching

Figure 7. Round gap fabrication process 


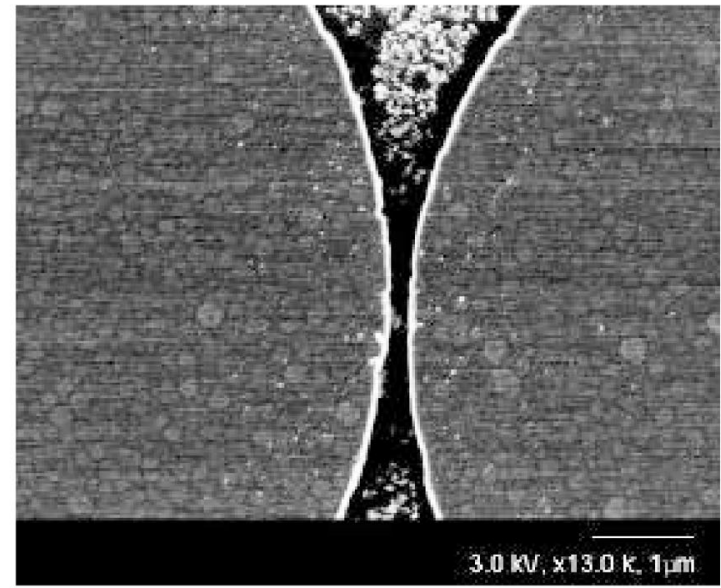

Figure 8. The round gap of $150 \mathrm{~nm}$

measured from the moment when the resistance of the Al bridge went to infinity. Figure 5 shows the relationship between the etching time after the gap opening and the gap size. Under 50 seconds of etching time, gap sizes smaller than $500 \mathrm{~nm}$ were obtained. The smallest sharp gap fabricated by this method was $20 \mathrm{~nm}$, and a 50nm gap is shown in Figure 6.

A round gap was also fabricated to test different types of electric fields. For example, a relatively straight electric field could be formed between the round electrodes for more highly ordered deposition of CNTs.

The round gap was fabricated with the pattern reversal of $\mathrm{Al}$ bridge shape as shown in Fig. 7. On top of the low stress $\mathrm{Si}_{3} \mathrm{~N}_{4}$

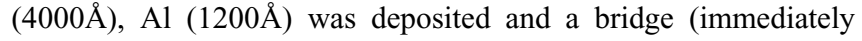
before a gap was formed) shape was created by the method described previously. Reactive ion etching (RIE) was used to undercut $\mathrm{Si}_{3} \mathrm{~N}_{4}$, and $\mathrm{Cr}(500 \AA)$ was then non-conformally deposited. The $\mathrm{Al}$ was removed together with the overlying $\mathrm{Cr}$ layer in $\mathrm{Al}$ etchant at $50^{\circ} \mathrm{C}$ (lift-off). Finally a round gap was created by the RIE of $\mathrm{Si}_{3} \mathrm{~N}_{4}$ and etching of $\mathrm{Si}$ in HNA $\left(\mathrm{HF}+\mathrm{HNO}_{3}+\mathrm{CH}_{3} \mathrm{COOH}\right)$.

The gap size was determined by the width of Al bridge prior to $\mathrm{Cr}$ deposition. The smallest gap size obtained by this method was $\sim 150 \mathrm{~nm}$, shown in Figure 8 . The depth of the trench between electrodes is $1.5 \mu \mathrm{m}$.

\section{CNT DEPOSITION}

For the assembly of CNTs over a gap, either a growth method or a deposition method can be used. A CVD growth method has been proposed to fabricate a free standing SWCNTs between the posts [9]. Recently, an electric field was applied to align CNTs during their growth on electrodes, since thermal vibration and gas flow can randomize the orientation of CNTs [8]. However, this method can limit the freedom of device fabrication by the growing conditions. Electrodes with high melting temperature metal were needed, and catalysts should be precisely patterned on top of electrodes. Also, specimens require extremely clean experimental conditions to prevent the generation of amorphous hydrocarbons.

On the other hand, CNT deposition can also be accomplished by dispersing CNTs in a solution and applying an electric field to attract CNTs between electrodes [10]. In the solution, CNTs as well as other particles may be attracted by a dipole moment, $p=q l$, where $q$ is electric charge and $l$ is the length of a CNT. However, it

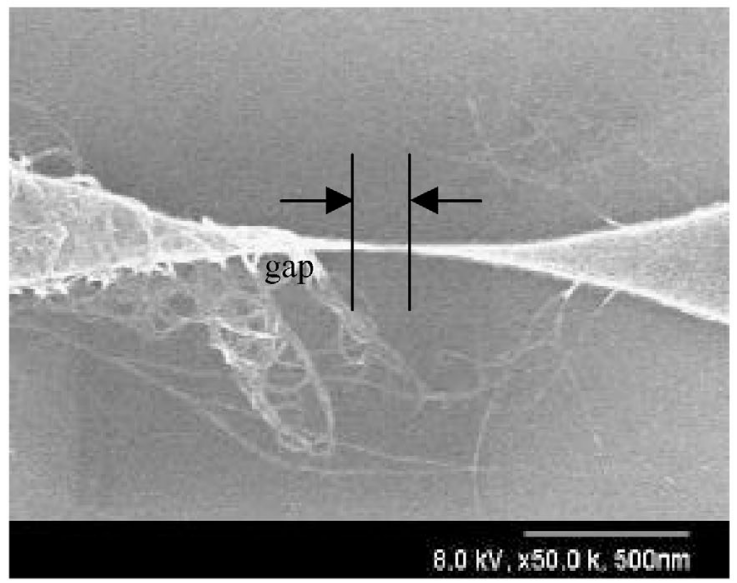

Figure 9. Carbon nanotube deposition in a sharp gap by $A C$ electrophoresis

was found that CNTs are slowly attracted by a DC electric field and many unwanted particles in the CNT solution are more easily deposited. CNTs could be easily attracted by a high-frequency $(\sim$ $5 \mathrm{MHz}) \mathrm{AC}$ field. In this case, round particles, such as catalysts and amorphous carbon debris, were not attracted, and could be excluded in the deposition process [11].

In our process, SWCNTs were dispersed and suspended in a dichloro-benzene solution. Subsequently, the solution was sonicated for several hours in order to untangle the CNT ropes. After sonication, one drop of CNT solution was placed on the gap and an electric field of $5 \mathrm{kV} / \mathrm{cm} @ 10 \mathrm{MHz}$ was applied. The electric field was applied until the solution was completely evaporated, and images were taken by scanning electron microscopy (SEM).

Figure 9 shows a bundle of entangled CNTs deposited across the sharp gap. Since a round electric field was formed around the electrodes, a bundle of CNTs was deposited along the electric field. Small particles were gathered by stronger electric fields between sharp electrodes. It is believed that the sharp gap is not suitable for CNT deposition, but is better for nanoscale particle deposition.

Figure 10 shows the deposited CNTs across the round gap. In this case, CNTs were deposited with a more regular pattern, and were suspended across the gap. Compared with CVD methods, the suggested deposition steps can be performed at a room temperature in an open environment, thus providing more process freedom. A more refined process to deposit a single CNT is being developed. Various configurations for device application and fundamental experiments can be conceived by the proposed method.

\section{CONCLUSION}

New techniques for fabricating nanoscale gaps based on micro-lithography were proposed. A sharp gap was fabricated by two-step wet etching of an Al thin film. A round gap was created by reversing an Al bridge pattern with lift-off. The smallest gaps fabricated by this method were $20 \mathrm{~nm}$ for a sharp gap and $150 \mathrm{~nm}$ for a round gap. The fabrication steps yielded consistent results.

CNTs dispersed in dichloro-benzene were deposited across the fabricated gaps by AC electrophoresis. A bundle of entangled CNTs were deposited across the sharp gap, and more ordered CNTs were deposited across the round gap. The proposed method can be used for various device applications and fundamental experiments. 


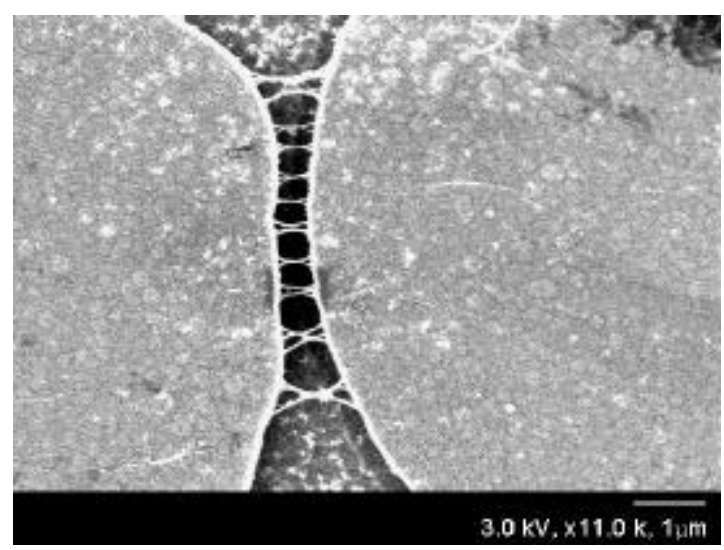

(a) top view

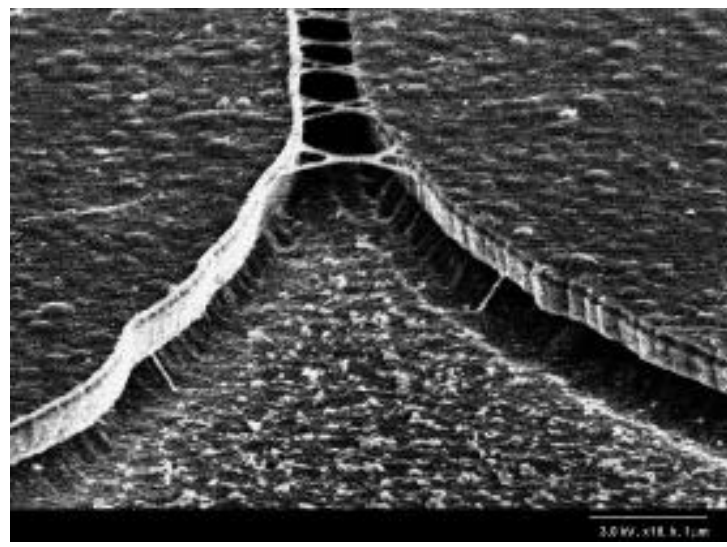

(b) side view

Figure 10. Carbon nanotube deposition on a round gap by $A C$ electrophoresis

\section{REFERENCES}

1. S. Iijima, "Helical microtubules of graphite carbon", Nature, 354, 56 (1994)
2. S. Akita, Y. Nakayama, S. Mizooka, Y. Tkano, T. Okawa, Y. Miyatake, S. Yamanaka, M. Tsuji, and T. Nosaka, "Nanotweezers consisting of carbon nanotubes operating in an automic force microscope", Appl. Phys. Lett. 79(11), 1691 (2001)

3. Y. Ren and D. L. Price, "Nuetron scattering study of $\mathrm{H}_{2}$ adsorption in single walled carbon nanotubes", Appl. Phys. Lett. 79, 3684 (2001)

4. P. G. Collins, K. Bradley, M. Ishigami and A. Zettl, "Extreme oxygen sensitivity of electronic properties of carbon nanotubes", Science 287, 1801 (2000)

5. J. Kong, N. R. Franklin, C. Zhou, M. G. Chopline, S. Peng, K. Cho and H. Dai, "Nanotube molecular wires as chemical sensors", Science, 287, 622 (2000)

6. M. F. Yu, O. Lourie, M. J. Dyer, K. Moloni, T. F. Kelly, and R. S. Ruoff, "Strength and Breaking Mechanism of Multiwalled Carbon Nanotubes Under Tensile Load", Science, 287, 637 (2000) 7. M. F. Yu, M. J. Dyer, G. D. Skidmore, H. W. Rohrs, X. K. Lu, K. D. Ausman, J. R. Von Ehr, and R. S. Ruoff, "Threedimensional manipulation of carbon nanotubes under a scanning electron microscope", Nanotechnology, 10, 244 (1999) 8. Y. Zhang, A. Chang, J. Cao, Q. Wang, W. Kim, Y. Li, N. Morris, E. Yenlimez, J. Kong, and H. Dai, "Electric-field-directed growth of aligned single-walled carbon nanotubes", Appl. Phys. Lett., 79(19), 3155 (2001)

9. A. M. Cassell, N. R. Franklin, T. W. Tombler, E. M. Chan, J. Han, and H. Dai, "Directed growth of free-standing single-walled carbon nanotubes", J. Am. Chem, Soc, 121, 7975 (1999)

10. K. Yamamoto, S. Akita and Y. Nakayama, "Orientation and purification of carbon nanotubes using ac electrophoresis", Appl. Phys. 31, L34, (1998)

11. X. Q. Chen, T. Saito, H. Yamada, and K. Matsushige, "Aligning single-wall carbon nanotubes with an alternating-current electric field”, Appl. Phys. Lett., 78(23), 3714 (2001)

\section{ACKNOWLEDGEMENT}

The authors appreciate support from the Office of Naval Research (ONR) Miniaturized Intelligent Sensors Program (MIS). 